\title{
Compostos funcionais presentes em bulbilhos de alhos armazenados sob refrigeração, provenientes de cultivos no Brasil e na China
}

\author{
Functional compounds present in garlic cloves stored under refrigeration from crops in Brazil and China
}

Ester Yoshie Yosino da Silva ${ }^{\text {I }}$ Celso Luiz Moretti ${ }^{I I}$ Leonora Mansur Mattos ${ }^{\text {II }}$

\section{RESUMO}

O objetivo desta pesquisa foi determinar o teor de compostos funcionais presentes em bulbilhos de alho das cultivares 'Caçador' e 'Peruano', originárias do Brasil, e da cultivar 'Jinxiang', proveniente da China, durante o armazenamento pós-colheita até sessenta dias. $O$ delineamento experimental utilizado foi o inteiramente casualizado, em esquema fatorial $3 \times 5$ (três cultivares de alho $x 5$ periodos de armazenagem), com três repetições, sendo a unidade experimental composta por 10 bulbos de alho. Os bulbos foram armazenados à temperatura de $22 \pm 1^{\circ} \mathrm{C}$ e umidade relativa de $70 \pm 2 \%$ e analisados quinzenalmente para teores de alicina, compostos fenólicos e atividade antioxidante pelo sistema $\beta$ caroteno/ácido linoléio. De maneira geral, verificou-se que as propriedades funcionais das cultivares de alho estudadas foram alteradas durante o armazenamento. O perfil dos teores de alicina não foi alterado no periodo de armazenamento para a cultivar 'Caçador'. Entretanto, o perfil foi de degradação do bioativo aos 45 e 15 dias de armazenamento para as cultivares 'Peruano' e 'Jinxiang', respectivamente. $O$ potencial antioxidante, medido pelo sistema $\beta$-caroteno/ácido linoléico para as cultivares 'Caçador' e 'Jinxiang', foi superior em relação à cultivar 'Peruano', no início da avaliação. O perfil para todas as cultivares foi de redução do potencial, sendo que, a partir dos 30 dias de avaliação, deixou-se de observar essas diferenças. Quanto ao conteúdo de compostos fenólicos totais, observaram-se maiores teores para a cultivar 'Jinxiang' em relação às cultivares 'Caçador' e 'Peruano' no início da avaliação. Todas as cultivares apresentaram aumento significativo a partir dos 15 dias de avaliação. Portanto, as cultivares de alho avaliadas possuem atividade antioxidante, proveniente dos compostos organossulfurados e compostos fenólicos, a qual foi alterada com o armazenamento refrigerado.
Palavras-chave: Allium sativum, alicina, compostos organossulfurados, atividade antioxidante, compostos fenólicos.

\begin{abstract}
The objective of the present research was to determine the content of functional compounds in garlic cloves of two Brazilian cultivars ('Caçador' and 'Peruano') one Chinese cultivar ('Jinxiang'), during the postharvest storage up to 60 days. The experiments were carried out in a completely randomized design, in a factorial scheme $3 \times 5$ (3 cultivars; 5 samplings times) with 3 replicates. Experimental unit was comprised by 10 garlic bulbs. Garlic bulbs were stored at $22 \pm 1^{\circ} \mathrm{C}$ and $70 \pm 2 \%$ RH and analyzed, every 15 days for allicin, phenolic compounds and antioxidant activity using the $\beta$-carotene/linoleic acid method. In general, it was observed that functional compounds were altered in the garlic cultivars studied. The profile of the levels of allicin was not changed during the period of storage for the variety 'Caçador'. However, the profile was of bioactive degradation at 45 and 15 days of storage for 'Peruano' and 'Jinxiang' cultivars, respectively. The antioxidant potential measured by the system $\beta$-carotene/ linoleic acid for cultivars 'Caçador' and 'Jinxiang' were higher than the 'Peruano' cultivar, at the beginning of the evaluation. The profile for all cultivars was of reduction of potential, and after 30 days these differences were no observed. Regarding the content of phenolic compounds it was observed that 'Jinxiang' showed higher levels than in the cultivars 'Peruano' and 'Caçador', at the beginning of the evaluation. All cultivars showed a significant increase from 15 days evaluation. Therefore, the garlic cultivars evaluated had antioxidant activity of compounds derived from organosulfur and phenolic compounds, which was modified with storage under refrigeration.
\end{abstract}

'Universidade de Brasília (UnB), Faculdade de Ciências da Saúde, Campus Universitário Darcy Ribeiro, 70910-900, Brasília, DF, Brasil. E-mail: esteryy@terra.com.br. Autor para correspondência.

"Embrapa Hortaliças, Ponte Alta-Gama, DF, Brasil. 
Key words: Allium sativum, allicin, organosulfur compounds, antioxidant activity, phenolics compounds.

\section{INTRODUÇÃO}

Alimento funcional é a denominação atribuída ao alimento que, além das suas funções nutricionais como fonte de energia e substrato para a formação de células e tecidos, possui, em sua composição, uma ou mais substâncias capazes de agir no sentido de modular os processos metabólicos, promovendo efeitos benéficos à saúde, podendo retardar o estabelecimento de doenças crônicodegenerativas e melhorar a qualidade e expectativa de vida das pessoas. Essas substâncias podem desempenhar funções, como antioxidantes, inibidores e indutores de enzimas, inibidores de atividades receptoras, indutores e inibidores da expressão de genes, entre outras. (SGARBIERI \& PACHECO, 1999; KRIS-ETHERTON et al., 2004).

Frutas e hortaliças representam excelentes fontes de compostos bioativos e seu consumo tem sido associado à redução de risco de muitas doenças crônicas, incluindo câncer, doenças cardiovasculares e inflamatórias (HANNUM, 2004). Esses alimentos têm sido altamente recomendados pela sua riqueza em vitamina C, carotenóides, substâncias fenólicas, substâncias sulfuradas, glicosídeos indólicos, frutooligossacarídeos, dentre muitos outros, com ação quelante e/ou redutora (SGARBIERI \& PACHECO, 1999).

O alho (Allium sativum L.) é uma hortaliça amplamente consumida em todo o mundo, cujas propriedades benéficas são reconhecidas há mais de 5000 anos (AMAGASE et al., 2001). Botanicamente, é classificado na família das liliáceas, que possui mais de 700 espécies, incluindo cebola, alho poró e cebolinha (HOLUB et al., 2002).

O alho contém 33 compostos organossulfurados (COS), sendo que $1 \mathrm{~g}$ de alho fresco contém de 11 a 35mg dessas substâncias; possui ainda quase quatro vezes mais $\operatorname{COS}$ (por grama de peso fresco) do que a cebola, brócolis, couve-flor e damasco (HOLUB et al., 2002). Dentre os compostos com elevado valor funcional nos bulbos, destaca-se a alicina (dialil tiossulfonato), um líquido volátil responsável pelo odor pungente do alho. Quimicamente, a alicina é uma molécula instável e altamente reativa. É o composto bioativo mais comum e representa cerca de $70 \%$ dos compostos sulfurados presentes nessa hortaliça (MIRON et al., 2004).

Quando os bulbos de alho são cortados ou esmagados, a alicina é produzida enzimaticamente pela interação do aminoácido não protéico aliina [(+)-S-allilL-sulfóxido cisteína], abundante nos dentes de alho, com a enzima aliinase. Durante a reação enzimática, amônio e piruvato também são formados. Elementos responsáveis pela quebra da alicina são o ar, a água e temperaturas elevadas (MIRON et al., 2002, 2004; BAGHALIAN et al., 2005; SANCE et al., 2006).

Os compostos bioativos presentes no alho têm sido amplamente reconhecidos como agentes de prevenção e tratamento de doenças cardiovasculares e outras doenças metabólitas como aterosclerose, hiperlipidemia, trombose, hipertensão e diabetes, além de possuírem uma variedade de efeitos biológicos como atividade antimicrobiana, antiinflamatória e anticâncer. Muitos desses efeitos podem estar relacionados às suas atividades antioxidantes (AGARWAL, 1996; MIRON et al., 2002)

Há uma grande variabilidade no conteúdo de alicina e de outros COS no alho. Tal conteúdo é dependente da cultivar, da composição do solo, condições climáticas, época de colheita e manuseio pós-colheita. Com relação à época de colheita $\mathrm{e}$ manuseio pós-colheita, a quantidade de aliina e $\gamma$ glutamilcisteínas presentes em bulbos de alho aumentam nas quatro semanas que antecedem a época da colheita. Durante o processo de cura (plantas secas a sombra ou ao sol por até duas semanas), essas substâncias aumentam em aproximadamente $25 \%$. Um atraso de duas semanas na colheita aumenta o conteúdo desses compostos, correspondendo a um adicional de $20 \%$ da massa, em base seca (HOLUB et al., 2002).

Apesar de todos os estudos desenvolvidos em várias partes do mundo envolvendo a cultura do alho, há uma lacuna na literatura consultada acerca do conteúdo de substâncias bioativas em bulbos de alho durante o armazenamento pós-colheita. HUGHES et al. (2006) indicaram claramente a necessidade de um maior aprofundamento nas pesquisas enfocando essa questão.

O presente trabalho teve como objetivo determinar as alterações nos principais compostos funcionais (teor de alicina, atividade antioxidante e compostos fenólicos totais) em bulbos de alho fresco produzidos no Brasil e na China e armazenados sob refrigeração durante sessenta dias.

\section{MATERIAL E MÉTODOS}

Bulbos de alho das cultivares brasileira ('Caçador' e 'Peruano') foram obtidos nos campos experimentais da Embrapa Hortaliças, Brasília, DF e os bulbos da cultivar 'Jinxiang', importada da China, foram 
adquiridos na CEASA-DF. Todos os materiais utilizados foram cultivados na safra 2008. Os materiais foram selecionados, classificados e armazenados em câmara fria a $22 \pm 1^{\circ} \mathrm{Ce} 70 \pm 2 \%$ de umidade relativa por 60 dias. A cada quinze dias, durante um período de sessenta dias, amostras dos bulbos foram retiradas para análises.

Para a determinação do conteúdo de alicina, foi preparado um extrato aquoso de alho. Bulbilhos de $(0,7 \mathrm{~g}$ a $0,9 \mathrm{~g})$ foram descascados, esmagados, colocados em tubos plásticos de $50 \mathrm{~mL}$ e deixados em repouso a temperatura ambiente por aproximadamente $6 \mathrm{~min}$ para que os COS fossem produzidos. Em seguida, foram adicionados $10 \mathrm{~mL}$ de água deionizada a temperatura ambiente e os tubos fechados, utilizando-se filme plástico (Parafilm), e colocados sob agitação por aproximadamente 30 segundos com o auxílio de um agitador de tubos (IKA ${ }^{\circledR}$, modelo MS1, Alemanha). Posteriormente, as amostras foram filtradas através de uma membrana filtrante de $0,45 \mu \mathrm{m}$ (Millipore) e transferidas para um frasco de cromatografia líquida de alta eficiência (CLAE).

A solução padrão de alicina foi obtida pela oxidação do dialil dissulfeto, segundo LAWSON \& WANG (2001). Para a curva de calibração, foram feitas leituras, em espectrofotômetro, nos comprimentos de onda de $240 \mathrm{~nm}$ e $254 \mathrm{~nm}$. O cálculo da concentração de alicina (C) na solução foi realizado de acordo com a seguinte equação:

$$
E_{1 c m}^{1 \%}=\frac{\text { Absorbância }}{C\left(\mu g m L^{-1}\right)} \times 10000 \text {, em que: }
$$

$E=$ coeficiente de extinção para alicina em água (145,4, considerando-se uma célula de $1 \mathrm{~cm}$ e comprimento de onda de $240 \mathrm{~nm}$ ).

Posteriormente, diluições foram feitas para as concentrações de $5,10,15,20,30,40,50,60,70$ e $80 \mu \mathrm{g} \mathrm{mL}^{-1} \mathrm{e}$ analisadas por CLAE, para a construção da curva padrão.

O conteúdo de alicina foi determinado de acordo com o método 110.001 do INSTITUTE FOR NUTRACEUTICAL ADVANCEMENT (2005). As análises foram conduzidas em cromatógrafo líquido de alta eficiência com coluna de fase reversa LC-18 (150x4,6mm, 5 $\mu \mathrm{m}$ ) da Shimadzu (Japão), equipado com uma bomba de gradiente 300psi LC, com módulo degasificante (DGU-M10A), válvula micro-injetora, detector UV de arranjo de iodo (SPD-M10A) com comprimento de onda de $240 \mathrm{~nm}$, injetor manual e automático de amostras (SIL-10AF) e forno (CTO-10A) com temperatura de $28 \pm 0,5^{\circ} \mathrm{C}$. As amostras foram eluídas com metanol e água, na proporção (50:50) ao fluxo de $1,0 \mathrm{~mL} \mathrm{~min}^{-1}$ por $20 \mathrm{~min}$. O volume de injeção foi de $25 \mu \mathrm{L}$.
O extrato etanólico de alho foi obtido para determinação da atividade antioxidante e de compostos fenólicos totais. Bulbilhos de aproximadamente $3 \mathrm{~g}$ foram descascados, esmagados, e colocados em tubos plásticos de $100 \mathrm{~mL}$, envolvidos por papel alumínio. A amostra foi então deixada em repouso por aproximadamente $6 \mathrm{~min}$ para que os COS fossem produzidos. Foram adicionados $20 \mathrm{~mL}$ de etanol a $70 \%$ e os tubos fechados com filme plástico (Parafilm) e colocados sob agitação em agitador orbital (Hitachi, modelo EFOU-KT, Japão) a $16800 \mathrm{~g}$ por $60 \mathrm{~min}$. Após esse período, os tubos foram colocados em banho ultrassônico (Odontobrás, modelo 1440 Plus, Brasil), por 20min. O extrato foi separado do resíduo pela filtração através de papel de filtro quantitativo. $\mathrm{O}$ resíduo foi novamente submetido ao processo de extração, acima descrito. Os dois filtrados foram combinados e o volume aferido para $50 \mathrm{~mL}$.

A avaliação da atividade antioxidante foi realizada pela oxidação acoplada do $\beta$-caroteno e ácido linoléico, segundo a metodologia descrita por AMIM \& TAN (2002), com modificações de ISMAIL et al. (2004). A solução de $\beta$-caroteno $(1 \mathrm{~mL})$ foi preparada pela dissolução de $2 \mathrm{mg}$ de $\beta$-caroteno em $10 \mathrm{~mL}$ de clorofórmio, sendo colocada em um balão de fundo redondo, contendo $0,02 \mathrm{~mL}$ de ácido linoléico e $0,2 \mathrm{~mL}$ de $100 \%$ Tween 20 . Após remoção do clorofórmio, em evaporador rotatório (Tecnal, modelo TE-20, Brasil) a $40^{\circ} \mathrm{C}$, a mistura foi imediatamente diluída em $100 \mathrm{~mL}$ de água destilada, para formar uma emulsão. Alíquotas $(5,0 \mathrm{~mL})$ dessa emulsão foram transferidas para uma série de tubos de ensaio contendo $0,2 \mathrm{~mL}$ do extrato etanólico de alho (amostra) e $0,2 \mathrm{~mL}$ de etanol $70 \%$ (controle). Um branco para cada amostra foi utilizado para comparação, consistindo de $0,2 \mathrm{~mL}$ da amostra e $5,0 \mathrm{~mL}$ da emulsão sem $\beta$-caroteno. Após ser homogeneizada, a leitura foi feita em espectrofotômetro (Hitachi, modelo U-1100, Japão) a 470nm, sendo esta a leitura no tempo zero (tempo inicial). Em seguida, os tubos foram colocados em banho-maria a $45^{\circ} \mathrm{C}$ por duas horas e a absorbância foi registrada a cada $15 \mathrm{~min}$.

A atividade antioxidante (AA) foi medida em termos do descoramento do $\beta$-caroteno e calculada pela seguinte equação:

$$
A A=\frac{(1-A c)}{A a m} \times 100, \text { em que }
$$

$\mathrm{Ac}=$ Absorbância $(\mathrm{Abs})$ controle, Aam=Absorbância da amostra e $A c=($ Abs inicial - Abs final $)$.

A determinação do conteúdo de compostos fenólicos totais das amostras de alho fresco foi realizada de acordo com o método espectrofotométrico descrito por SINGLETON \& ROSSI (1965), modificado por 
NUUTILA et al. (2003), utilizando o reagente FolinCiocalteu, adquirido da Sigma-Aldrich (Alemanha). Foram adicionados em tubos de ensaio $400 \mu \mathrm{L}$ do extrato etanólico de alho, $400 \mu \mathrm{L}$ de etanol $70 \%, 400 \mu \mathrm{L}$ de FolinCiocalteu e $2000 \mu \mathrm{L}$ da solução saturada de carbonato de sódio $20 \%$. Os tubos foram homogeneizados, utilizando um agitador de tubos (IKA ${ }^{\circledR}$, modelo MS1, Alemanha). Posteriormente, foram adicionados mais $800 \mu \mathrm{L}$ da solução de carbonato de sódio $20 \%$. As amostras foram centrifugadas (centrífuga Sorvall ${ }^{\circledR}$, rotor SS-34, modelo RC6 Plus, Estados Unidos) por $3 \mathrm{~min}$ a $23450 \mathrm{~g}$ e mantidas por $20 \mathrm{~min}$ à temperatura ambiente. A leitura da absorbância foi medida em espectrofotômetro (Hitachi, modelo U100, Japão) com comprimento de onda de $735 \mathrm{~nm}$. O padrão da curva foi obtido utilizando-se ácido gálico em seis concentrações diferentes $\left(0,005\right.$ a $\left.0,03 \mathrm{mg} \mathrm{mL}^{-1}\right)$. Os resultados obtidos foram expressos em equivalentes de ácido gálico (EAG) $100 \mathrm{~g}^{-1}$ de matéria seca.

O delineamento experimental utilizado foi o inteiramente casualizado, em esquema fatorial $3 \times 5$ (três cultivares de alho $\mathrm{x}$ cinco períodos de armazenamento), com três repetições. A unidade experimental foi composta por 10 bulbos de alho. Os dados obtidos foram submetidos à análise de variância (ANOVA) e a comparação entre as médias dos resultados foi realizada pelo teste de Tukey, ao nível de 5\% de significância $(\mathrm{P} \leq 0,05)$.

\section{RESULTADOS E DISCUSSÃO}

Os teores de alicina da cultivar 'Caçador', durante 60 dias de armazenamento refrigerado, mantiveram-se estatisticamente inalterados. Por outro lado, houve redução significativa para as cultivares 'Jinxiang' e 'Peruano', a partir dos 15 e 45 dias de estocagem, respectivamente. Verificou-se que não houve diferença significativa quanto ao teor de alicina, entre as cultivares analisadas, até os 15 dias de armazenamento. A partir dos 30 dias, as cultivares mostraram perfis significativamente diferentes (Figura 1A).

Dentre as cultivares avaliadas neste estudo, a cultivar 'Jinxiang' apresentou maior perda do teor de alicina durante o armazenamento. Isso se deve provavelmente ao armazenamento pós-colheita, pois esses produtos chegam ao Brasil e são armazenados em câmara fria à temperatura $<-3^{\circ} \mathrm{C}$ para serem, então, comercializados, local em que são mantidos à temperatura de refrigeração até o momento de venda para o consumidor. Os estudos conduzidos por ICHIKAWA et al. (2006) mostram que os níveis de aliina não foram alterados com o armazenamento em temperaturas controladas, tendo em vista que bulbos de alho da cultivar 'Fukuchi-howarito', produzidos no Japão e armazenados em temperatura de $-3^{\circ} \mathrm{C}$ durante 150 dias, não apresentaram alterações nos conteúdos de aliina. Esse resultado indica também que os teores de alicina não foram alterados, já que esse composto é produzido enzimaticamente a partir do aminoácido não protéico aliina com a enzima aliinase, no momento que os bulbilhos são cortados. Já em temperaturas não controladas, como verificado neste estudo para a cultivar 'Jinxing', a bioquímica do alho pode ter sido alterada pela destruição parcial ou total dos precursores da alicina, inativação parcial ou total da enzima aliinase e perda dos compostos nutricionais (LAGUNAS \& CASTAIGNE, 2008).

A literatura cita somente resultados do teor de alicina referentes a bulbos de alho armazenados à temperatura ambiente (aproximadamente $25^{\circ} \mathrm{C}$ ). Dentre esses trabalhos pode-se citar o estudo conduzido por EAGLING \& STERLING (2000), que utilizaram a variedade Australiana Branca, produzida na Austrália e armazenada por 52 dias. Os resultados mostraram variação nos níveis de alicina no decorrer da pesquisa, sendo o valor médio de $6,1 \mathrm{mg} \mathrm{g}^{-1}$, com um máximo de $6,9 \mathrm{mg} \mathrm{g}^{-1}$ e um mínimo de $5,6 \mathrm{mg} \mathrm{g}^{-1}$. Não foram verificadas perdas no teor de alicina durante os 52 dias de armazenamento.

Com relação à atividade antioxidante, medida pelo sistema $\beta$-caroteno/ácido linoléico houve redução significativa durante o período de armazenamento. Tendo-se verificado diferença significativa nos tempos 0 e 15 dias de estocagem. As cultivares 'Caçador' e 'Jinxiang' apresentaram maior atividade antioxidante em relação à cultivar 'Peruano' no início da pesquisa (Figura 1B).

Esse resultado difere dos estudos conduzidos por QUEIROZ et al. (2009). Eles avaliaram a atividade antioxidante em alho fresco nacional, armazenado à temperatura ambiente por 60 dias, utilizando o sistema $\beta$-caroteno/ácido linoléico, segundo o método de MILLER (1971). Os autores verificaram aumento significativo da atividade antioxidante durante o período de armazenamento, sendo os valores no início do estudo de $35,74 \pm 3,65 \% \mathrm{e}$ no final do experimento de $44,97 \pm 2,06 \%$. KEVERS et al. (2007), avaliando a capacidade antioxidante durante a estocagem de frutas e hortaliças, incluindo o alho armazenado à temperatura ambiente durante 30 dias, citam que houve aumento superior a $50 \%$ da capacidade antioxidante, quando determinada pelo método de sequestro de radicais DPPH (2,2-difenil -1- 


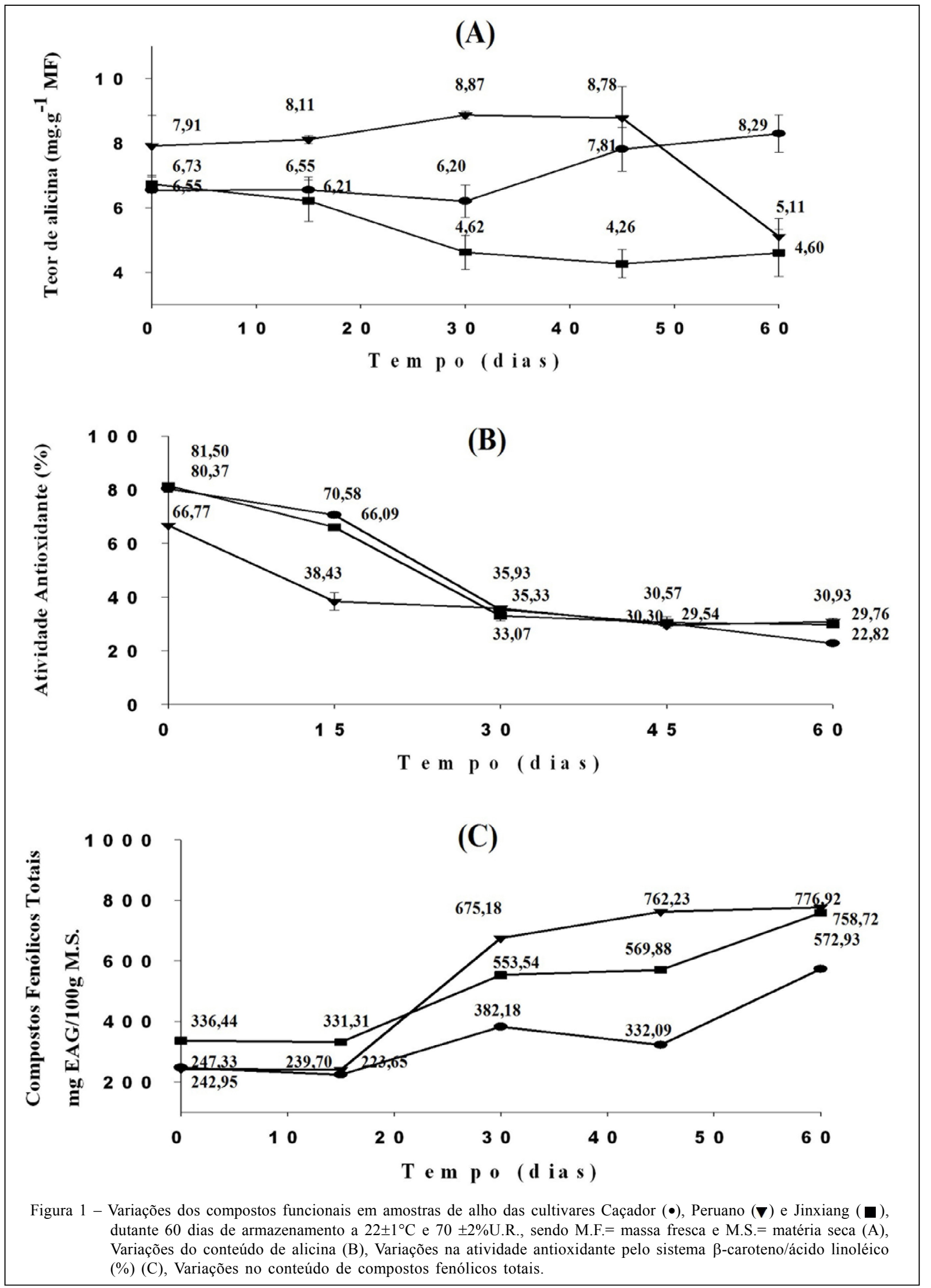

Ciência Rural, v.40, n.12, dez, 2010. 
picrilhidrazila). Os resultados contraditórios podem provavelmente ser devido a diferenças nas condições experimentais (diferentes mecanismos de reações) utilizados em diferentes testes, além das cultivares analisadas, condições climáticas e composição do solo, conforme também observado por HOLUB et al.(2002) e BOZIN et al. (2008).

$\mathrm{Na}$ determinação de compostos fenólicos totais (Figura 1C), todas as cultivares apresentaram aumento significativo a partir dos 15 dias de avaliação. Verificou-se que houve diferença significativa em todos os tempos de armazenamento, sendo que nos tempos 0 e 15 dias a cultivar 'Jinxiang' foi superior às cultivares 'Caçador' e 'Peruano'; aos 30 e 45 dias, a cultivar 'Peruano' foi superior às cultivares 'Caçador' e 'Jinxiang'; e, aos 60 dias, a cultivar 'Caçador' foi inferior às demais cultivares.

O perfil de fenólicos totais obtidos neste estudo diferiu dos obtidos por QUEIROZ et al. (2009). Eles verificaram aumento significativo a partir dos 30 dias de armazenamento à temperatura ambiente, sendo que tal tendência se manteve até aos 60 dias. Os teores de compostos fenólicos totais encontrados por aqueles pesquisadores foram de 6,99 $\pm 0,39 \mu \mathrm{g}$ de EAG $\mathrm{mg}^{-1} \mathrm{de}$ matéria seca no início do estudo, 7,23 $\pm 0,33 \mu \mathrm{g}$ de EAG $\mathrm{mg}^{-1}$ de matéria seca aos 30 dias e de $8,70 \pm 1,83 \mu \mathrm{g}$ de EAG mg ${ }^{-1}$ de matéria seca aos 60 dias, sendo superiores aos valores encontrados neste estudo. Outros relatos indicam que a contribuição de polifenóis totais como potencial antioxidante no alho fresco e possíveis reduções dependem da composição e quantidade de elementos minerais que participam no complexo de polifenóis, principal antioxidante da dieta (BENKEBLIA, 2005; GORISTEIN et al., 2006). Portanto, as diferenças encontradas entre os estudos aqui citados podem estar também diretamente relacionadas com a cultivar e condições de cultivo.

A atividade antioxidante no alho tem sido atribuída, principalmente, à presença de compostos contendo enxofre e polifenóis (NUUTILA et al., 2003; BENKEBLIA, 2005; GORISTEIN et al., 2006), mas poucos estudos mostram o potencial antioxidante do alho, durante a estocagem. Verificou-se neste trabalho que o alho cultivado e comercializado no Brasil apresenta atividade antioxidante e que é alterado com $\mathrm{o}$ armazenamento refrigerado.

\section{CONCLUSÃO}

Houve alteração significativa para as propriedades funcionais do alho durante os 60 dias de armazenamento refrigerado e diferenças significativas entre as cultivares cultivadas e comercializadas no mercado nacional ('Caçador', 'Peruano' e 'Jinxiang'). O resultado do conteúdo de alicina apresentou melhor estabilidade para a cultivar 'Caçador'. As cultivares avaliadas ('Caçador', 'Peruano' e 'Jinxiang') possuem propriedades antioxidantes, as quais são influenciadas pelo período de armazenamento. Para o conteúdo de compostos fenólicos totais, o maior conteúdo foi para a cultivar 'Jinxiang' no início do estudo e, a partir dos 30 dias de armazenamento, todas as cultivares apresentaram aumento significativo em seus teores.

\section{AGRADECIMENTOS}

À Coordenação de Aperfeiçoamento de Pessoal de Nível Superior - CAPES, pela concessão da bolsa de estudos e à Embrapa pelo financiamento da pesquisa.

\section{REFERÊNCIAS}

AGARWAL, K.C. Therapeutic actions of garlic constituents. Medicinal Research Reviews, Rhode Island, v.16, n.1, p.111124, 1996. Disponível em: <http://onlinelibrary.wiley.com/ doi/10.1002/(SICI)1098-1128(199601)16:1\%3C111::AID-ME D4\%3E3.0.CO;2-5/pdf>. Acesso em: 15 ago. 2009. doi: $10.1002 /($ SICI) $1098-1128$ (199601)16:1<111::AIDMED4>3.0.CO;2-5.

AMAGASE, $\mathrm{H}$. et al. Intake of garlic and its bioactive components. Journal of Nutrition, Bethesda, v.131, p.S955S962, 2001. Disponível em: <http://jn.nutrition.org/cgi/ reprint/ 131/3/955S >. Acesso em: 15 ago. 2009. doi: 0022-3166/01.

AMIM, I.; TAN, S.H. Antioxidant activity of commercial selected seaweeds. Malaysian Journal of Nutrition, Malaysa, v.8, n.2, p.167-177, 2002. Disponível em: <http:// psasir.upm.edu. my/ 7099/1/ mjn8n2_art4.pdf $>$. Acesso em: 15 ago. 2009.

BAGHALIAN,K. et al. Evaluation of allicin content and botanical traits in Iranian garlic (Allium sativum L.) ecotypes. Scientia Horticulturae, Netherlands, v.103, n.2, p.155166, 2005. Disponível em: <http://www.sciencedirect.com/ science? ob=MImg\& imagekey=B6TC3-4DWVY7B-1C\&_cdi $=5159 \&$ user $=687355 \&$ pii $=$ S0304423804001086\&_origin $=$ search\&_zone $=$ rslt_list_item\&_coverDate $=01 \% 2 \mathrm{~F}$ $01 \% 2$ F $2005 \&$ \& s $=998969997 \& \mathrm{wchp}=\mathrm{dGLbV1b}-$ zSkWb\&md5=3a3f299162ac59c38aa9e6c965f3d022\&ie $=/$ sdarticle.pdf>. Acesso em: 20 ago. 2009. doi:10.1016/ j.scienta.2004.07.001.

BENKEBLIA, N. Free-radical scavenging capacity and antioxidant properties of some selected onions (Allium cepa L.) and garlic (Allium sativum L.) extracts. Brazilian Archives of Biology and Technology, Curitiba, v.48, n.5, p.753-759, 2005. Disponível em: <http://www.scielo.br/pdf/ babt/v48n5/26611.pdf>. Acesso em: 20 ago. 2009. doi: 10.1590/S1516-89132005000600011.

BOZIN, B. et al. Phenolics as antioxidants in garlic (Allium sativum L., Alliaceae). Food Chemistry, Netherlands, v.111, p.925-929, 2008. Disponível em: $<\mathrm{http}: / /$ www.sciencedirect.com/ science?_ob=MImg\&_imagekey=B6T6R-4SFS0S7-9-7\&_cdi=

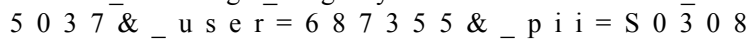


81460800530 X\&_orign $=$ search\&_coverDate $=12$ $\% 2 \mathrm{~F} 15 \% 2 \mathrm{~F} 2008 \&$ \&k $=998889995 \& \mathrm{view}=\mathrm{c} \& \mathrm{wchp}=\mathrm{dGLzVzz}-$ zSkWb\&md5=e34ba04ebf7a4bd373a5 5b08a18c9c6d\&ie=/ sdarticle.pdf>. Acesso em: 20 ago. 2009. doi:10.1016/ j.foodchem. 2008.04.071.

EAGLING, D.; STERLING, S. A cholesterol-lowering extract from garlic. Austrália: Rural Industries Research and Development Corporation, 2000. 18p.

GORISTEIN, S. et al. Dose-dependent influence of commercial garlic (Allium sativum) on rats fed cholesterol-containing diet. Journal of Agricultural and Food Chemistry, Washington, v.54, p.4022-4027, 2006. Disponível em: < http://pubs.acs.org/ doi/ pdfplus/10.1021/jf058182\%2B >. Acesso em: 20 ago. 2009. doi: $10.1021 / \mathrm{jf058182+}$.

HANNUM, S.M. Potential impact of strawberries on human health: a review of the science. Critical Reviews in Food Science and Nutrition, Philadelphia, v.44, p.1-17, 2004.

HOLUB, B.J. et al. Organosulfur compounds from garlic. In: SHI, J. et al. Functional foods: biochemical and processing aspects. Whashington:CRC, 2002. Cap.7, p.213-238.

HUGHES, J. et al. Effect of low storage temperature on some of the flavour precursors in garlic (Allium Sativum). Plant Foods for Human Nutrition, Netherlands, v.61, p.81-85, 2006. Disponível em: <http://www.springerlink.com/ content/ 045pm77 168gj2j05 / fulltext. pdf $>$. Acesso em: 20 ago. 2009. doi: $10.1007 / \mathrm{s} 11130-006-0018-4$.

ICHKAWA, M. et al. Changes in organosulfur compounds in garlic cloves during storage. Journal of Agricultural and Food Chemistry, Washington, v.54, p.4849-4854, 2006. Disponível em: <http://pubs.acs.org/doi/pdfplus/10.1021/ jf060083o $>$. Acesso em: 15 ago. 2009. doi:10.1021/jf060083o.

INSTITUTE FOR NUTRACEUTICAL ADVANCEMENT. Allicin content in garlic by HPLC (INA method 110.001). NSF INTERNATIONAL; Allicin by high-performance liquid chromatography, INA Method 110.001, 2005. Capturado em: 15 jan. 2008. Online. Disponível em: 'http:// www.nsf.org/business/ina/garlic.asp.

ISMAIL, A. et al. Total antioxidant activity and phenolic content in selected vegetables. Food Chemistry, Netherlands, v.87, p.581-586, 2004. Disponível em: $<\mathrm{ht} \mathrm{t}$ p: // w w w. s c i e n c e d i rect. c o m / science?_ob=MImg\&_imagekey=B6T6R-4BS4F4C-1$\mathrm{R} \& \&_{\text {c }} \mathrm{c}$ d i $=5037$ \&_ u s e r $=6877355$ \&_pii $=\overline{\mathrm{S}} 0308814604000366 \&$ origin $=$ search $\&$ coverDate $=10 \% 2 \mathrm{~F} 31$ $\% 2 \mathrm{~F} 2004 \&$ sk $=999129995 \& \mathrm{view}=\mathrm{c} \& \mathrm{w} \mathrm{chp}=\mathrm{dGLbVzz}-$ zSkzV\&md5=7f3f50dda20b22622a 4d7d9d77c63d20\&ie $=/$ sdarticle.pdf>. Acesso em: 20 ago. 2009. doi:10.1016/ j.foodchem. 2004.01.010.

KERVERS, C. et al. Evolution of antioxidant capacity during storage of selected fruits and vegetables. Journal of Agricultural and Food Chemistry, Washington, v.55, p.8596-8603, 2007. Disponível em: <http://pubs.acs.org/doi/ pdfplus/10.1021/jf071736j>. Acesso em: 20 ago. 2009. doi: $10.1021 / \mathrm{jf} 071736 \mathrm{j}$.

KRIS-ETHERTON, P.M et al. Bioactive compounds in nutrition and health-research methodologies for establishing biological function: the antioxidant and anti-inflammatory effects of flavonoids on atherosclerosis. Annual Reviews of Nutrition, Califórnia, v.24, p.511-538, 2004.

LAGUNAS, L.L.M.; CASTAIGNE, F. Effect of temperature cycling on allinase activity in garlic. Food Chemistry, Netherlands, v.111, p.56-60, 2008. Disponível em: <http://www.sciencedirect.com/ science?_ob=MImg\&_imagekey=B6T6R-4S2MJ18-7-F\&_cdi $=5037 \& \_$user $=687355 \& \_$pii $=\mathrm{S} 0308814608003257 \& \_$origin $=$search \& coverDate $=11 \% 2 \mathrm{~F} 01 \% 2 \mathrm{~F} 2008 \&$ sk $=99888999$

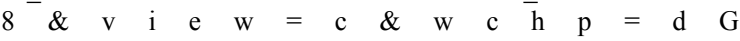
LzVtzzSkWb\&md5=84cee378eac2b854d1a14f66bc40d32e\&ie=/ sdarticle.pdf $>$. Acesso em: 15 ago. 2009. doi:10.1016/j.foodchem. 2008.03.035.

LAWSON, L.D.; WANG, Z.J. Low allicin release from garlic supplements: a major problem due to the sensitivities of alliinase activity. Journal of Agricultural and Food Chemistry, Washington, v.49, p.2592-2599, 2001. Disponível em: <http:/ /pubs.acs.org/doi/pdfplus/ 10.1021/jf001287m>. Acesso em: 20 ago. 2009. doi:10.1021/jf001287m.

MILLER, H.E. A simplified method for the evaluation of antioxidants. Journal of the American Oil Chemists' Society, United States, v.48, p.91, 1971.

MIRON, T. et al. [H] Allicin: preparation and applications. Analytical Biochemistry, United States, v.331, p.364-369, 2004. Disponível em: <http://www.sciencedirect.com/ science?_ob=MImg\&_imagekey=B6W9V-4CDHFYY-1-1\&_c $\mathrm{di}=6692 \&$ user $=687 \overline{3}$ 55\& pii $=\mathrm{S} 000326 \quad 9704003069 \&$ origi $\bar{n}=\mathrm{se}$ a r c h \& c over D a te $=08 \% 2 \mathrm{~F} 15$ $\overline{\%} 2 \mathrm{~F} 2004 \&$ sk $=996689997 \& \mathrm{view}=\mathrm{c} \& \mathrm{wchp}=\mathrm{dGLbVtz}-$ zSkzV\&md5=6 ffcd6f $1 \mathrm{c} 413$ bd559f $40 \mathrm{~b} 0 \mathrm{ab} 46 \mathrm{c} 1 \mathrm{a} 460 \& \mathrm{ie}=/$ sdarticle.pdf>. Acesso em: 20 ago. 2009. doi:10.1016/j.ab.2004. 03.054 .

MIRON, T. et al. A spectrophotometric assay for allicin, alliin, and alliinase (alliin lyase) with a chromogenic thiol: reaction of 4mercaptopyridine with thiosulfinates. Analytical Biochemistry, United States, v.307, n.1, p.76-83, 2002. Disponível em: <http:/ $/ \mathrm{w}$ w w science?_ob=MImg\&_imagekey=B6W9V-46BSFYH-C 3K\& cdi $=66 \overline{9} 2$ \&_user $=687355 \&$ \& ii $=$ S 0003269702000 $106 \&$ \&origin $=$ search\&_coverDate $=08 \% 2 \mathrm{~F} 01 \%$ 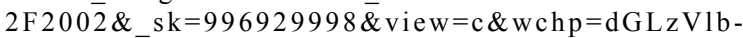 zSkWA\&md $5=829 \mathrm{c} 0638 \mathrm{be} 4 \mathrm{~b} 47 \mathrm{db} 689 \mathrm{~d} 557 \mathrm{~d} 55 \mathrm{ced} 2$ 7e\&ie $=/$ sdarticle.pdf $>$. Acesso em: 15 ago. 2009. doi: 10.1016/S00032697(02)00010-6.

NUUTILA, A.M. et al. Comparison of antioxidant activities of onion and garlic extracts by inhibition of lipid peroxidation and radical scavenging activity. Food Chemistry, Netherlands, v.81, p.485-493, 2003. Disponível em: <http:// w w w . s c i e n c e d i r e c t. c o m / science?_ob=MImg\&_imagekey=B6T6R-47PPB3V-Y7\&_cdi $=5037 \&$ user $=687355 \&$ pii $=$ S 0308814602004764 $\& \quad$ or ig i n $=\mathrm{s} \mathrm{e} \mathrm{a} \mathrm{r} \mathrm{c} \mathrm{h} \mathrm{\&} \mathrm{c} \mathrm{o} \mathrm{v} \mathrm{e} \mathrm{r} \mathrm{D} \mathrm{a} \mathrm{t} \mathrm{e}=06 \% 2$ F30\%2F2003\&_sk=999189995 \&view $=\mathrm{c} \& \mathrm{wchp}=\mathrm{dGLbVtb}$ zSkzS\&md5 $=512942381514620 \mathrm{fe} 63 \mathrm{e} 371 \mathrm{db} 0745726 \& \mathrm{ie}=/$ sdarticle.pdf>. Acesso em 20 ago. 2009. doi: 10.1016/S03088146(02)00476-4.

QUEIROZ, Y.S. et al. Garlic (Allium sativum L.) and readyto-eat garlic products: in vitro antioxidant activity. Food Chemistry, Netherlands, v.115, p.371-374, 2009. Disponível em: <http://www.sciencedirect.com/ 
science?_ob=MImg\&_imagekey=B6T6R-4V74VMK-1 $5 \&$ cd $\mathrm{i}=5037 \&$ user $=687355 \&$ pii $=\mathrm{S} 03088146080$ $14374 \&$ origin $=$ search\&_cover Date $=07 \% 2 \mathrm{~F} 01$ $\% 2$ F $2009 \&$ sk $=998849998 \&$ view $=c \& w c h p=d G L$ bVtzzSkzk\&md5=7ddd9fb1465543722a71184fcd4f691e\&ie=/ sdarticle.pdf>. Acesso em: 15 ago. 2009. doi:10.1016/ j.foodchem.2008.1 1.105.

SANCE, M.M. et al. Evaluation of the Argentinean garlic germplasm in relation to its aptitude for the freeze drying process. Molecular Medicinal Chemistry, Argentina, v.10, p.33-34, 2006
SGARBIERI, V.C.; PACHECO, M.T.B. Revisão: alimentos funcionais fisiológicos. Brazilian Journal of Food Technology, Campinas, v.2, p.7-19, 1999. Disponível em: < http://www.ital.sp.gov.br/ bj/artigos/bjft/1999/p9902.pdf >. Acesso em: 20 ago. 2009.

SINGLETON, V.L.; ROSSI, J.A. Colorimetry of total phenolics with phosphomolybdic-phosphotungstic acid reagents. American Journal of Enology and Viticulture, Davis, v.16, p.144-158, 1965 . 